\title{
A GAUSSIAN PROCESS MODEL FOR TIRES IN COMBINED SLIP CASE
}

\author{
André Pelisser ${ }^{1}$, Alexandre Kawamoto ${ }^{1}$, Wilhelm Vatanabe ${ }^{2}$, Victor Namba ${ }^{2}$ \\ ${ }^{1}$ ETAS \\ ${ }^{2}$ Escola Politécnica da Universidade de São Paulo \\ E-mails: andre.pelisser@etas.com, alexandre.kawamoto@etas.com, \\ wilhelm.andriessen@usp.br, victor.namba@usp.br
}

\begin{abstract}
A deep knowledge of tire behavior in operating conditions is fundamental to the effective modelling of vehicular dynamics on its safety, comfort and performance aspects. Data-based models are a common approach despite the associated challenges: the complex interaction between tire constructive and operational factors implies the necessity of large datasets, the tradeoff between local and global fit is challenging and the handling of a high number of inputs with varying relevance to each output is a computationally expensive problem.

A very promising approach to data-based modelling is the Gaussian Process Regression (GPR), a class of supervised learning. Data points are used to train an underlying probability distribution with characteristics assumed a priori. The resulting model has relatively small requirement of training data, robustness against overfitting, good response to complex behavior and computational tractability.

The aim of this work is to support the elaboration of data-based tire models by creating one of a Formula SAE specific 10" slick tire. Procedures are presented for the use of GPR to fit the data locally and then predictions are made on lateral and longitudinal forces with respect to vertical load, slip-angle, slip-ratio, pressure and camber. In the end, model quality metrics will be established for internal cross validation and comparison to test data.
\end{abstract}

\section{INTRODUCTION}

Tire behavior is governed by complex non-linear interactions between different factors, making the process of modelling lateral and longitudinal forces with precision a challenging task. Nevertheless, many applications require easy to handle, computationally simple and at the same time very accurate models.

Over the years, different approaches of tire modeling have been developed, tuned for the specificities and needs of different applications. Salvagni et al (2013) [1] lists models ranging from finite elements method to brush models, shell models, and experimental multiparametric polynomial interpolations. Pacejka [2] groups this broad variety in four major categories: 
1. Pure empirical model: experimental data regression whose parameters are obtained exclusively from the training data.

2. Semi-empirical: assumptions are made on the expected model response characteristics and tendencies. Experimental data is used to determine some key fitting parameters for the specific tire analyzed. E.g. Brush model tire mechanics (Svendenius, Gäfvert 2005).

3. Simple physical model: simple model based on physical assumptions, generally limited to a simple condition, as pure slip. E.g. Thin walled involucres (Salvagni et al, 2013) [1].

4. Complex physical models: highly complex physical formulations valid for a broader range of conditions. They are usually challenging to build and computationally demanding to evaluate. E.g. Finite elements method (Nakashima, Wong, 1993).

General aspects of each one of these categories are presented in figure 1

Figure 1: Comparison of tire model aspects according to different approaches

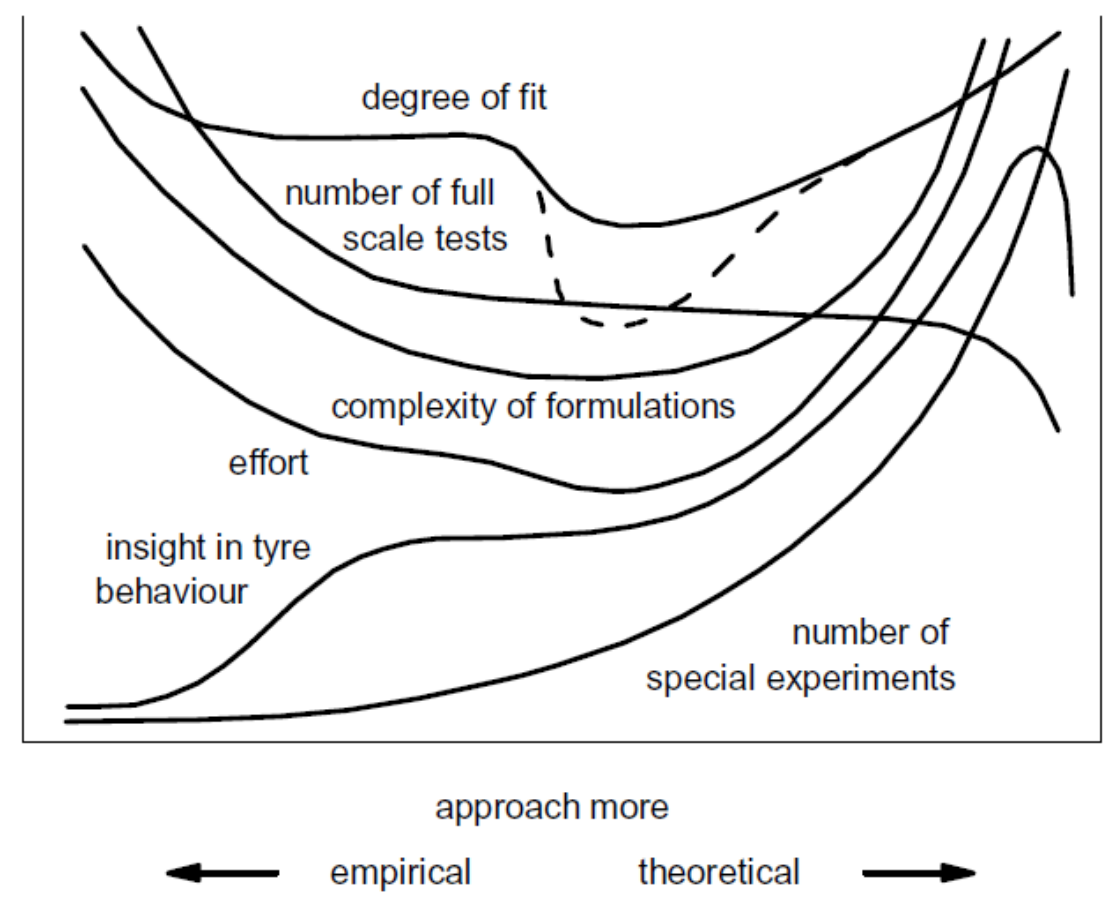

Source: Pacejka (2006) - Tire and vehicle dynamics, p. 85, Figure 2.11

In this text, tire behavior is determined by applying Gaussian process regression over a set of empirical training data using ETAS Advanced Simulation for Calibration, Modeling and Optimization (ASCMO). A review of Bayesian modelling technics using Gaussian process is presented in the next section to give an insight on ASCMO internal methods.

This paper presents an approach to determine lateral and longitudinal tire forces in a combined slip case accounting for vertical load, camber and inflation pressure. The resulting multivariate model is continuous, computationally light and requires only a small amount of training data. The predicted forces are compared to measured data and a Friction Ellipse is shown. 


\section{MODELING METHODOLOGY}

Supervised learning methods may be categorized into two common approaches [3]:

1. Using a specific class of functions as base for modeling. It has the drawback of resulting in poor model prediction capabilities if the chosen function set does not represent well the phenomena being analyzed. Expanding the richness of the considered class functions may result in overfitting, where the fit to training data is satisfactory but it fails to make reliable predictions about generic test cases.

2. Giving a prior probability to every possible function, assigning higher probabilities to functions considered more likely. At first glance, this proposal appears to be unfeasible as there are infinity sets of possible functions. Nonetheless, there are methods to work out this challenge and generate the desired predictions in a reasonable amount of time.

\subsection{Bayesian Modelling Overview}

The second approach presented is referred as a Bayesian method [3]. It tackles the regression problem (mapping a multivariate input vector $x$ to a continuous output predictive function $f(x)$ ) using the following logic: Firstly, a prior distribution is defined containing characteristics expected of the system behavior. It is then combined to a given dataset of $\mathrm{n}$ observations of the phenomena $D_{n x 1}=\left\{\left(x_{i}, f_{i}\right) \mid i=1, \ldots, n\right\}$, resulting in a posterior distribution. From this posterior, it is possible to synthesize functions that predict the system response to the inputs.

To illustrate this with a simple example, a single input case is presented. Figure 2 shows four possible predictive functions drawn from the priori (left panel) and the resulting posteriori by combining it with two datapoints (right panel). The dashed functions represent four possible posteriori samples and the continuous line the overall mean, which is the model prediction.

Figure 2: Example of four random samples of a prior distribution (left) and the resulting posterior distribution after the conditioning to two training points (right)
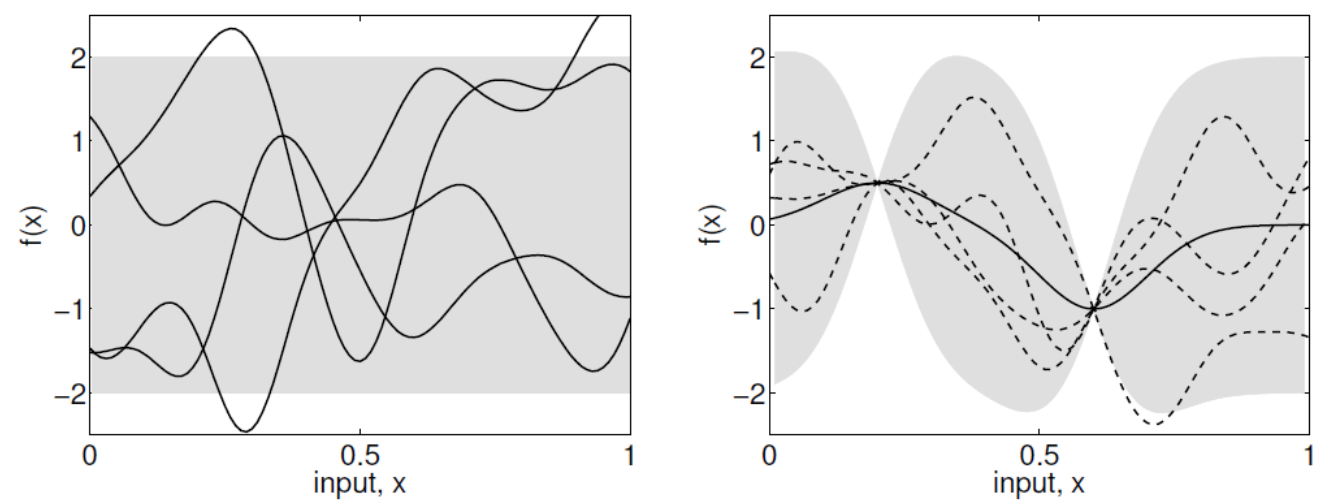

Source: (2006) - Gaussian Processes for Machine Learning, p. 3, Figure 1.1

It is important to notice that the posteriori here was constructed in a way that all the sample functions passes through the training datapoints, which implies the assumption of perfect accuracy of the data acquisition process. It is possible as well to construct models that accounts for the inaccuracy of data. Finally, the shaded regions represent twice the local standard deviation for each input $\mathrm{x}$, which highlights another advantage of Bayesian modelling: the prediction is accompanied by an estimate of its "precision". 


\subsection{Bayesian Modelling using Gaussian process}

This paper uses ETAS ASCMO platform for modeling, which relies on a specific class of Bayesian models denominated Gaussian process models. A Gaussian process is defined as a collection of random indexed variables (stochastic process) such that every finite collection of those random variables has a multivariate normal distribution. A key property of such process is that they can be completely determined by its mean function $m(x)$ and covariance function $k\left(x, x^{\prime}\right)[3]$

The random variables represent the value of the function $f(x)$ at location $x$ :

$$
\begin{aligned}
& f(x) \sim \mathcal{G P}\left(m(x), k\left(x, x^{\prime}\right)\right), \text { where } \\
& m(x)=\mathbb{E}[f(x)] \\
& k\left(x, x^{\prime}\right)=\mathbb{E}\left[(f(x)-m(x))\left(f\left(x^{\prime}\right)-m\left(x^{\prime}\right)\right)\right]
\end{aligned}
$$

\subsection{1 - Covariance function properties and definition}

The modeling technic presented in this paper uses a Gaussian process distribution as the prior probability distribution. Therefore, the choice of the covariance function is crucial in synthesizing a suitable Gaussian process as it encodes our assumptions about the expected model behavior. ASCMO allows the usage of the traditional Squared Exponential (SE) or, alternatively, Matérn covariance functions. The proposed model on this paper employs the first, defined by equation (4) for two generic points $p, q$ :

$$
\operatorname{cov}\left(f\left(x_{p}\right), f\left(x_{q}\right)\right)=k\left(x_{p}, x_{q}\right)=\exp \left(-\frac{1}{2}\left|x_{p}-x_{q}\right|^{2}\right)
$$

The squared exponential class of functions have the desirable properties of being infinitely differentiable and also stationery/isotropic as the covariance between two output points $f\left(x_{p}\right), f\left(x_{q}\right)$ is a function of $\left|x_{p}-x_{q}\right|$ and therefore invariant to translations or any other "rigid motion" on the input space. They are also called radial basis functions since $k\left(x_{p}, x_{q}\right)$ is only a function of $r=\left|x_{p}-x_{q}\right|$. Its value is close to the unity for variables whose inputs are very close to each other and decreases with the distance. [3]

Some free parameters called hyperparameters are included in the covariance function in order to account for different behavior and uncertainty level present in training data. The length-scale $\mathrm{L}$ divides the $\left|x_{p}-x_{q}\right|$ term and represents, informally, how far you need to move in input space for the function values to become uncorrelated. On its turn, the signal variance $\sigma_{f}^{2}$ is a pre-factor that controls the overall variance of the random function. Their combination results in:

$$
\operatorname{cov}\left(f\left(x_{p}\right), f\left(x_{q}\right)\right)=k\left(x_{p}, x_{q}\right)=\sigma_{f}^{2} \exp \left(-\frac{1}{2} \frac{\left|x_{p}-x_{q}\right|^{2}}{L^{2}}\right), \text { where }
$$

$\mathrm{L}$ is the characteristic length scale $\sigma_{f}^{2}$ is the signal variance 
Additionally, for "real life" modelling applications, available data usually does not represent the exact value of the measured variable, but an approximation limited by the acquisition process accuracy. This is taken into account by assuming additive, independent and identically distributed Gaussian noise $\varepsilon$ with variance $\sigma_{n}^{2}$ acting over each training output:

$$
\begin{aligned}
& y_{i}=f\left(x_{i}\right)+\varepsilon, \text { where } \\
& \varepsilon \sim \mathcal{N}\left(0, \sigma_{n}^{2}\right)
\end{aligned}
$$

The resulting covariation function for the output affected by Gaussian noise is:

$$
\operatorname{cov}\left(y\left(x_{p}\right), y\left(x_{q}\right)\right)=k_{y}\left(x_{p}, x_{q}\right)+\sigma_{n}^{2} \delta_{p q}=\sigma_{f}^{2} \exp \left(-\frac{1}{2} \frac{\left|x_{p}-x_{q}\right|^{2}}{L^{2}}\right)+\sigma_{n}^{2} \delta_{p q}, \text { where }
$$

$\sigma_{n}^{2}$ is the noise variance

$\delta_{p q}$ is the Kronecker delta: $\left\{\begin{array}{l}1 \text { if } p=q \\ 0 \text { if } p \neq q\end{array}\right.$

In order to illustrate the impact of varying the hyperparameters on model results and quality an example is presented:

Figure 3 - Influence of hyperparameters on a GP Model
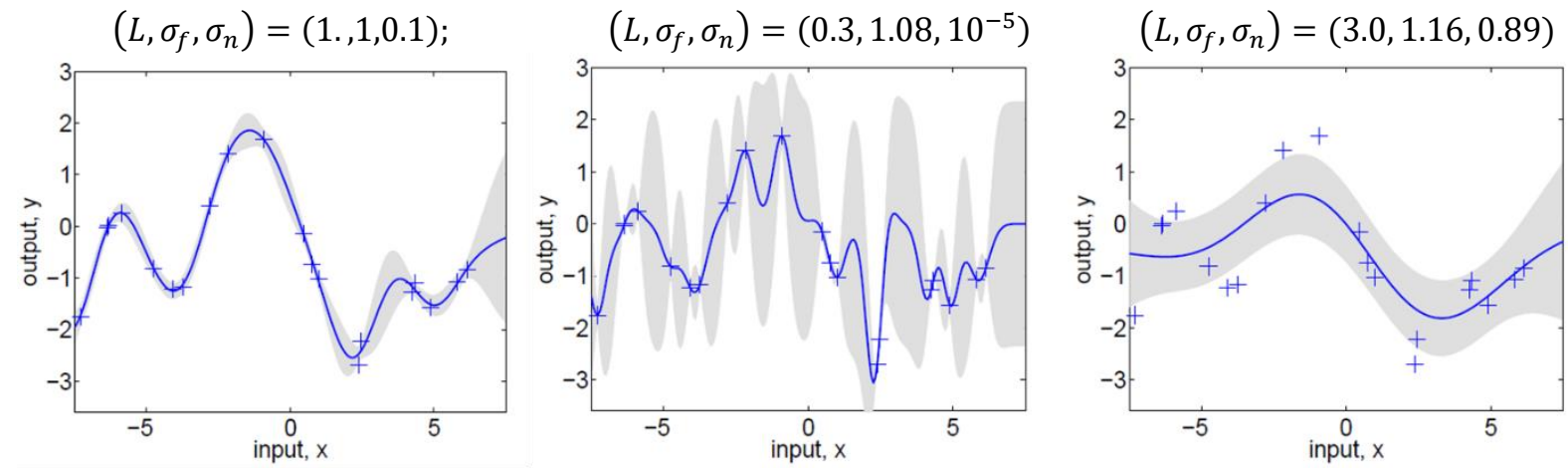

Source: (2006) - Gaussian Processes for Machine Learning, p. 20, Figure 2.5

The leftmost panel presents an optimal model (blue line) with $\left(L, \sigma_{f}, \sigma_{n}\right)=(1 ., 1,0.1)$ and a $95 \%$ confidence zone shaded in gray. In the middle panel, length-scale was reduced to 0.3 and the other parameters set by optimizing marginal likelihood. The model now has a lot more flexibility for "explaining" de data locally as it gives much less importance to the influence of distant points. This results in $\sigma_{n}=0.00005$, an extremely low value, as the model fits data by a quickly varying signal with low noise. On the other hand, the rightmost panel model has a higher length-scale of 3, and interprets data as a slowly varying signal with very high noise level $\sigma_{n}=0.89$. The balance between these two descriptions is achieved by optimizing marginal likelihood while setting the covariance function parameters.

\subsection{2 - Posterior determination and model generation}

It is possible to construct a covariance matrix for a set of $\mathrm{n}$ training points applying equation (8) element-wise: 


$$
\begin{aligned}
& \operatorname{cov}(y)=K(X, X)+\sigma_{n}^{2} I, \text { where } \\
& \mathrm{X}=\{(x i) \mid i=1, \ldots, n\} \\
& \mathrm{y}=\{(y i) \mid i=1, \ldots, n\}
\end{aligned}
$$

We define now a function $f_{*}=f\left(x i_{*}\right)$ which predicts outputs for a given $n_{*}$-dimensional test dataset $\left\{\left(x i_{*}\right) \mid i=1, \ldots, n_{*}\right\}$. The joint distribution of the training outputs $y$ and test outputs $f_{*}$, according to the previously defined prior and assuming mean $m(x)=0$ is:

$$
\left[\begin{array}{l}
y \\
f_{*}
\end{array}\right] \sim \mathcal{N}\left(0,\left[\begin{array}{cc}
K(X, X)+\sigma_{n}^{2} I & K\left(X, X_{*}\right) \\
K\left(X_{*}, X\right) & K\left(X_{*}, X_{*}\right)
\end{array}\right]\right)
$$

At this point, creating the model corresponds to conditioning the joint Gaussian prior distribution on the observations: $f_{*} \mid X_{*}, X, y$, a simple operation [3].This corresponds, in other words, to adding the "knowledge" provided by the data observed to the expected model characteristics defined by the covariance function. The result is a joint posterior distribution containing all the information needed for value prediction and also the associated model variance. It is convenient to define $\bar{f}_{*}$ as the mean of the posteriori distribution, which is them assumed to correspond to the "most likely behavior" for the outputs giving the training inputs and the prior.

$$
\begin{aligned}
& f_{*} \mid X_{*}, X, y \sim \mathcal{N}\left(\bar{f}_{*}, \operatorname{cov}\left(f_{*}\right)\right), \text { where } \\
& \bar{f}_{*} \triangleq E\left[f_{*} \mid X_{*}, X, y\right]=K\left(X_{*}, X\right)\left[K(X, X)+\sigma_{n}^{2} I\right]^{-1} y, \\
& \operatorname{cov}\left(f_{*}\right)=K\left(X_{*}, X_{*}\right)-K\left(X_{*}, X\right)\left[K(X, X)+\sigma_{n}^{2} I\right]^{-1} K\left(X, X_{*}\right)
\end{aligned}
$$

The next step is predicting an output $\bar{f}\left(x_{*}\right)$ at one specific test point $x_{*}$. The n dimensional vector of covariance between a test point and $\mathrm{n}$ training points is defined:

$$
k\left(x_{*}, X\right)=\left\{k\left(x_{*}, x_{i}\right) \mid i=1, \ldots, n\right\}=k_{*}
$$

Applying equations $(\mathrm{x})$ and $(\mathrm{y})$ to test point $x_{*}$ using $k_{*}$ notation results in:

$$
\begin{aligned}
& \bar{f}\left(x_{*}\right)=k_{*}^{T}\left(K+\sigma_{n}^{2} I\right)^{-1} y \\
& \mathbb{V}\left[\bar{f}\left(x_{*}\right)\right]=k\left(x_{*}, x_{*}\right)-k_{*}^{T}\left(K+\sigma_{n}^{2} I\right)^{-1} k_{*}
\end{aligned}
$$

Expanding equation $(\mathrm{x}$ ) for in a matrix notation for more clarity results in:

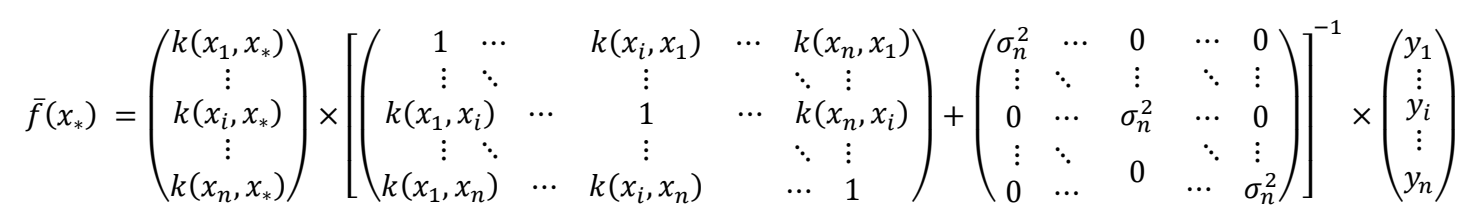

ASCMO formulation rewrites equation (15) to present it as a linear combination of $n$ kernel functions, each one centered on training point: 


$$
\begin{aligned}
& \bar{f}\left(x_{*}\right)=\sum_{i=1}^{n} \alpha \cdot k\left(x_{i}, x_{*}\right) \text { where }, \\
& \alpha=\left(K+\sigma_{n}^{2} I\right)^{-1} y
\end{aligned}
$$

\section{DATA GENERATION AND PRE-PROCESSING}

\subsection{Tire force generation mechanisms overview}

Some of the most important components in vehicle design are the tires. Firstly, tires have to support the vehicle weight, aerodynamic forces, and road banking. Furthermore, they are the primary source of force and moment generation, which affects vehicle behavior in many different ways. A complete understanding of tire behavior and all the factors that affect it are fundamental to effectively provide comfort, control, and stability to the vehicle being designed.

"Print" or "footprint" are the names given to the area of the tread of a tire that is in direct contact with the ground. The rubber patch contained in the print is either stuck to the ground or sliding across the road. Tire forces normally are generated by a combination of two factors: friction/sliding between the print and the road and elastic deformations on the tread and the structures of the tire due to rubber adhesion to the ground.

A variety of mechanisms, including mechanical grip to the texture of the pavement and molecular adhesion to the surface, is responsible for sticking the rubber to the road. When the sliding condition is reached, the force generated continues to depend on vertical load, rubber and pavement characteristics and begins to be influenced by relative sliding velocity [4].

Due to the complexity of tire behavior, it is usual to analyze each one of its characteristics separately, keeping most factors fixed while varying only a few parameters. Lateral and longitudinal forces are commonly studied individually as the combined case is highly nonlinear and complex and, therefore, difficult to be understood and modeled.

According to SAE J670, figure 4 - left, the lateral force originates at the center of the tire contact to the ground and it is perpendicular to the plane of the wheel. The standard also defines the slip angle, $\alpha$, which has a fundamental connection to lateral force generation. Figure 4 - right shows a typical plot for lateral force versus slip angle for a racing tire. The curve was divided into three sections: elastic or linear, in which the print is stuck on the road and the lateral force is produced by elastic deformations; frictional, where the print is predominantly sliding across the pavement; transitional, where we have a combination of frictional and elastic effects. 
Figure 4 - SAE J670 standard; Typical lateral force versus slip angle plot
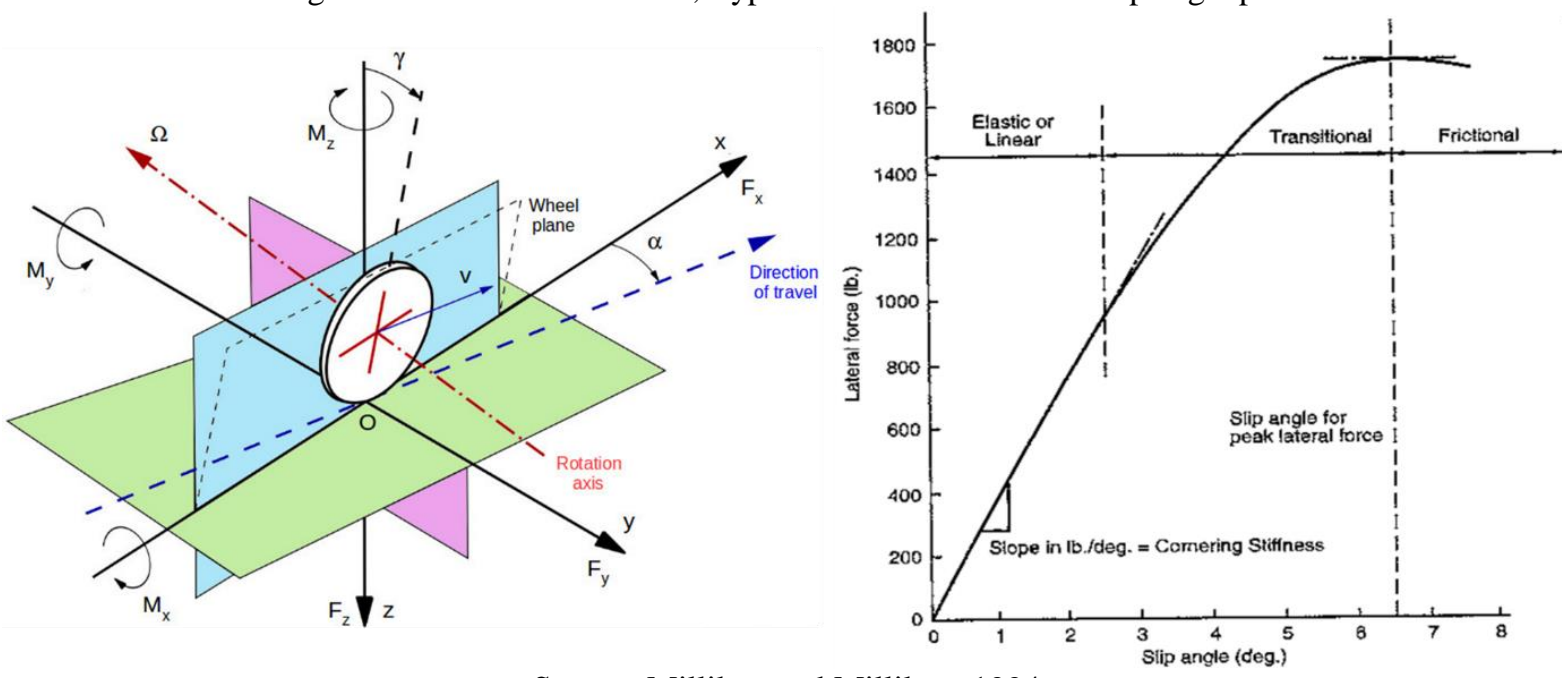

Source: Milliken and Milliken, 1994

SAE J670 also defines the slip ratio as $\mathrm{SR}=\left(\Omega-\Omega_{0}\right) / \Omega_{0}$, where $\Omega$ is the angular velocity of the driven wheel and $\Omega_{0}$ is the angular velocity of the free-rolling situation. Just like the lateral forces, longitudinal forces originate at the center of the tire contact to the pavement. The force direction, however, is indicated by the vector Fx on figure 4. Typical plots for longitudinal forces are shown in figures 5 . Traction and braking behavior are noticeably different due to subtle changes in the force generation mechanism. As in the lateral case, we have regions where each of the presented mechanisms - friction, elastic and transitional - predominates.

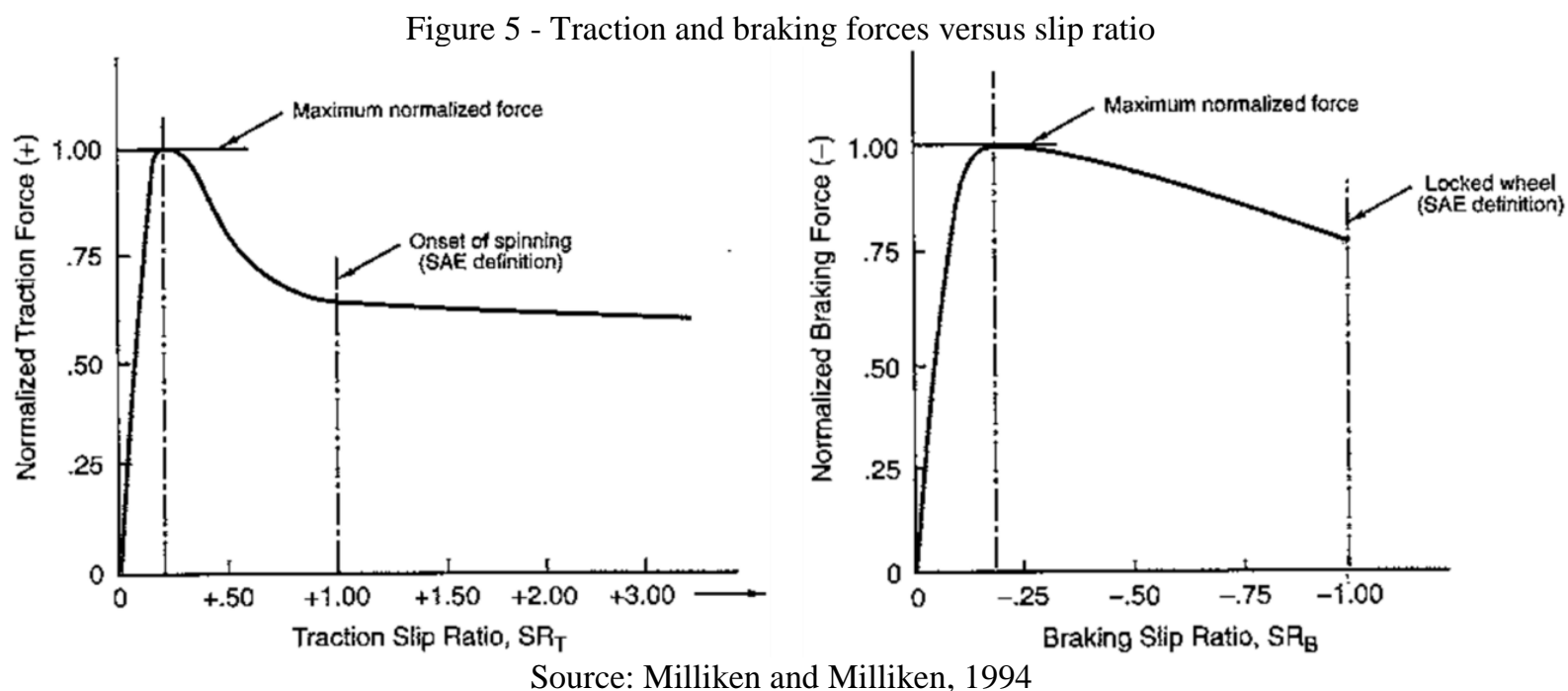

In a combined operation case, when the tire is producing both lateral and longitudinal forces, the mechanisms involved end up competing against each other, especially when the friction/sliding factor becomes dominant. In other words, for high values of slip angle, the tire's longitudinal force generation capability is reduced, as the sliding condition induced by high slip angles hinders the generation of longitudinal force by the elastic mechanism. An analogous situation occurs for high values of slip ratio and lateral force.

The main variables responsible for force generation on tires - slip angle and slip ratio -were briefly described above. However, there are many other factors that significantly affect tire behavior, altering both shape and size of the curves shown above. Temperature, inflation 
pressure, vertical load, camber, road characteristics, tire compound are some examples of which variables should be considered in the elaboration of models that aim to describe these phenomena. This large number of relevant parameters, associated with the complex interactions that occur between them, makes tire modeling a very difficult and challenging task.

\subsection{Tire Test Consortium installations and test procedure}

In order to feed the mathematical model described previously (section 1) with reliable data, tire test results from Formula SAE Tire Test Consortium (FSAE TTC) were used. FSAE TTC is a consortium designed to pool resources from Formula SAE teams and employ them in testing some of the most commonly used tires in the category. Testing facilities are provided by Calspan, a renowned company in the area. Accurate data on tire behavior is essential for the teams to develop their projects correctly, which makes the activity exerted by FSAE TTC fundamental to the development of the category and its participants.

The machine used for FSAE TTC tire tests is shown in figure 6. When initially built, it was the world's first high speed, high load, six-component flat-belt tire testing machine and remains the most capable tire testing machine in the world. The capability at Calspan Tire Research Facility (TIRF) is recognized worldwide, as customers include all forms of professional auto racing (Formula 1, NASCAR, Champ Car/IRL, etc.) [5]

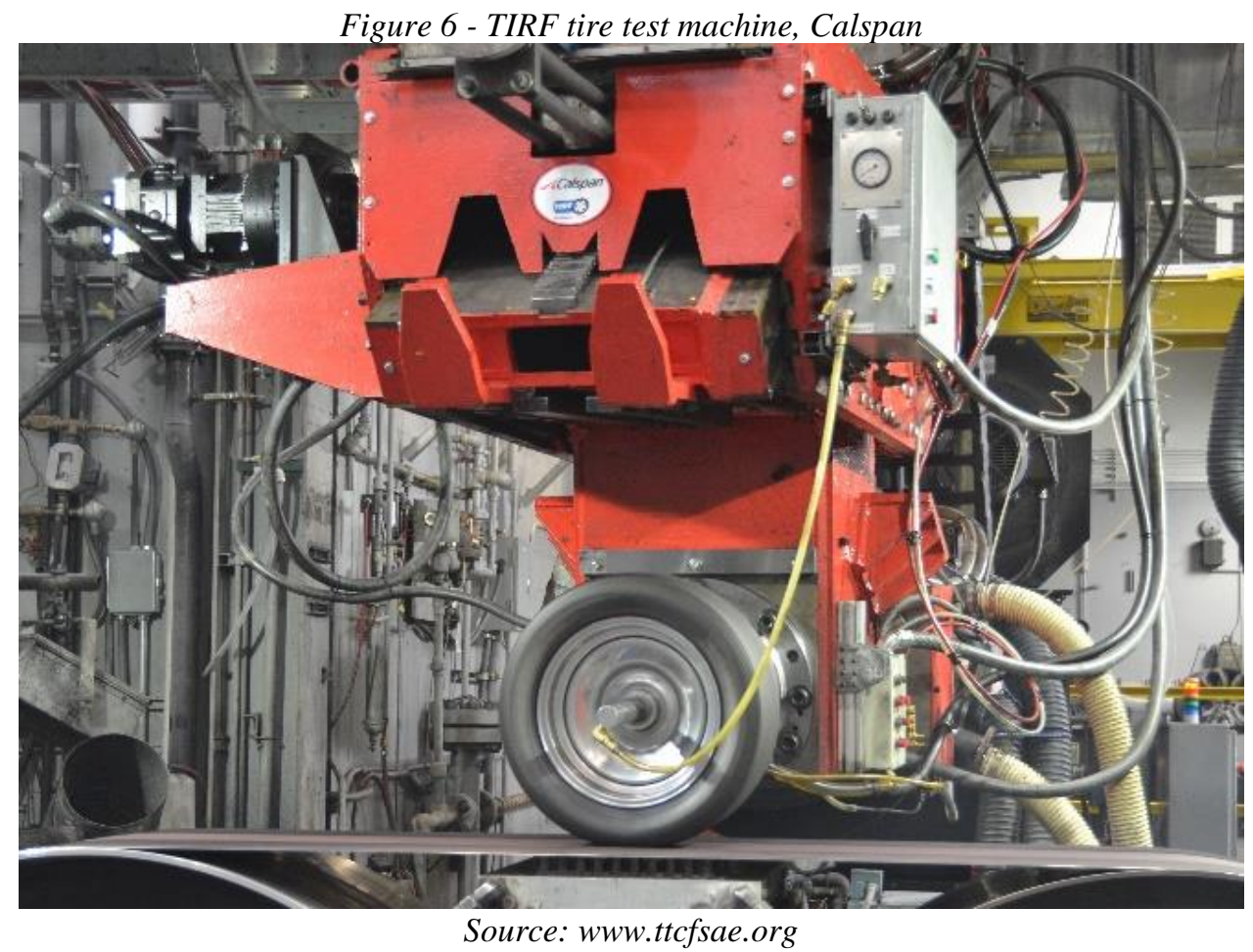

Each test run measures over time a set of relevant variables to tire behavior, amongst which we can mention, slip angle, slip ratio, "road" speed, wheel rotational speed, temperature in different parts of the tire, etc. The test parameters were adjusted to FSAE conditions. Vertical load, camber, and inflation pressure are some examples.

The test runs are divided into three main parts: cornering, drive/brake/combined and transient. In the cornering testing, the slip ratio is kept at zero, while the other parameters, including the 
slip angle, are varied in the stipulated range. In the drive/brake/combined test, the slip angle is held constant while the other parameters are swept. The term "combined" is used to describe simultaneous non-zero slip angles and slip ratios. Transient test includes simultaneous variations in parameters such as road speed and slip angle, aiming to observe the tire's transient response.

\subsection{Model inputs range and definition}

With the measured data in hand, some preliminary models were generated using different combinations of input and output variables. The most relevant parameters in tire performance analysis were chosen as inputs to build these models: inflation pressure, tire temperature, camber, vertical load, slip angle and slip ratio. The main objective was to predict lateral and longitudinal forces, which were chosen as output variables.

Tire temperature, a very useful and important parameter in tire performance, was not considered in the final model. In the test data, it behaves more like an output variable - being a result of variations in the other input variables - than as an input and so was not included.

Another important parameter is road speed. In the tests, some values of road speed $(15,25,45$ $\mathrm{mph}$ ) were used at specific moments, e.g. at transient and drive/brake conditions. However, most of the test data was acquired at $25 \mathrm{mph}$ - chiefly the desired combined slip cases -, which made natural for us to choose only that velocity.

The other five parameters are described below:

- Inflation pressure (P): Air pressure inside the tire. In the tests, its value is kept constant at 8 psi (55 kPa), 10 psi (69 kPa), 12 psi $(83 \mathrm{kPa})$ or 14 psi $(97 \mathrm{kPa})$.

- Camber or Inclination Angle (IA): Inclination angle of wheel plane relative to road plane. The values used in the test were $0,1,2,3$ and 4 degrees. These are the most common camber angles used in Formula SAE cars.

- Vertical Load (FZ): Vertical force applied to the tire. Most FSAE cars weigh between 170 $\mathrm{kg}$ to $300 \mathrm{~kg}$. Considering these range of weigh and the load transfer, the vertical load used in the test ranges from $200 \mathrm{~N}$ to $1100 \mathrm{~N}$.

- Slip Angle (SA): Defined by SAE J670, slip angles values ranged from -12 degree to 12 degree, selected to ensure that the peak of lateral force was surpassed.

- Slip Ratio (SR): The slip ratio measured in the test follows the Calspan definition. $\mathrm{SR}=0$ does not imply FX $=0$. Similar to what was done for the slip angle, slip ratio range was selected to surpass the longitudinal force peak, -0.20 to 0.15 .

The raw data containing all these variables as a function of time has several parts that are not useful for the proposed combined slip model. Measurement errors and transient instabilities found in some more sensible variables, such as slip ratio, might spoil the mathematical model. Spring rate and transient tests contain information that will not be analyzed in this model. Additionally, warm up section presents undesired temperature variations. All of these unwanted points were excluded from both training and test dataset. 


\subsection{Data reduction and training points definition}

In total 567.077 measure points were available in the complete dataset provided by FSAE TTC, which must initially be divided into two independent subsets: training data (used to train the model) or test data (used as validation). Based on previous experiences with complex models, ETAS suggests the usage of 200 to 2000 points as training data size for a 5-input model. To reduce data size from over 500.000 points to less than 2.000, ASCMO uses a random selection algorithm, ensuring there is no bias.

In order to assess the balance between model quality and complexity, a sensitivity analysis was conducted over training data size. The model size was increased by 50 from an initial value of 100 points up to 1200 . The evaluation metric chosen was the root mean square of "test error". It corresponds to the difference between model prediction and measured value (for all test data $\sim 500.000$ points) for both lateral and longitudinal forces. Figure (7) shows the root mean square error versus training data size plot for lateral and longitudinal forces.

Figure 7 - Root mean square error versus training data size
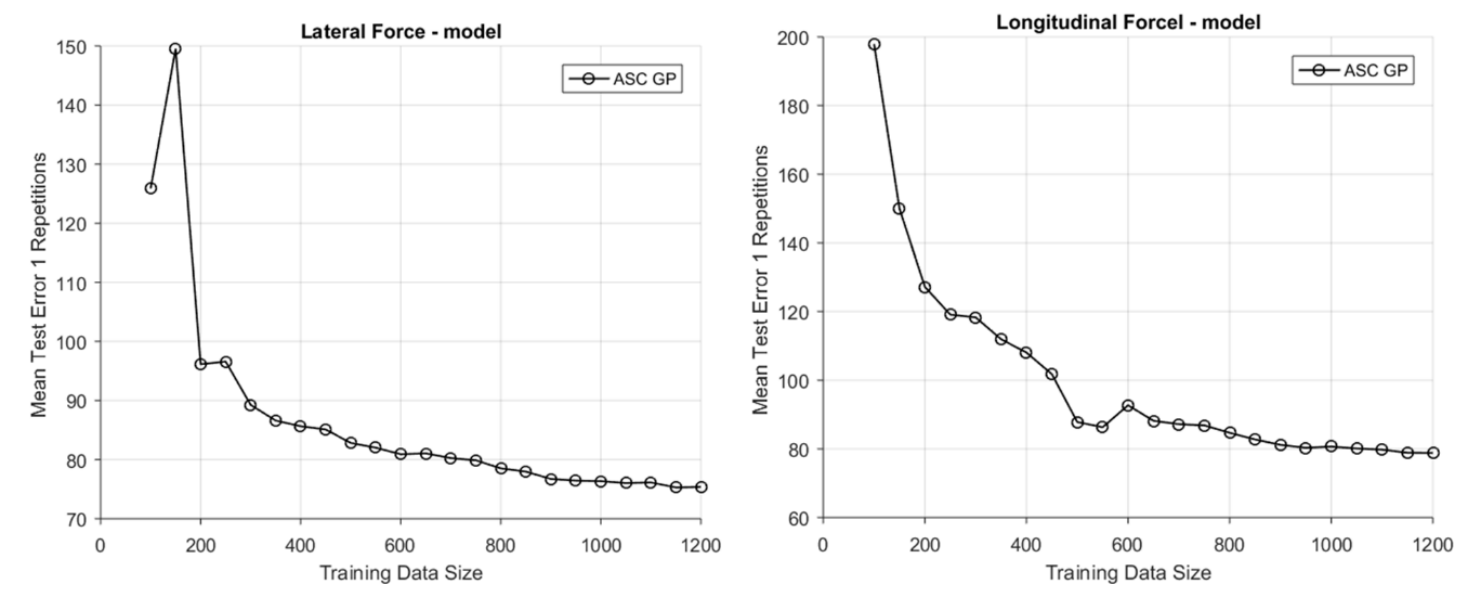

Source: The authors - ETAS ASCMO Environment

It is natural that more training points implies in a higher point density in the n-dimensional input space resulting in better models and smaller errors. On the other hand, every additional training point generates a new kernel function as described in equation (17) adding computational cost to model evaluation. Based on the results presented in figure 7 , a set of 800 training points was elected to guarantee the balance between model versatility and precision. The scatter plots in the figures (8) to (12) illustrate, for each variable, a comparison between the original distribution (test data plus training data) and the 800 points training data:
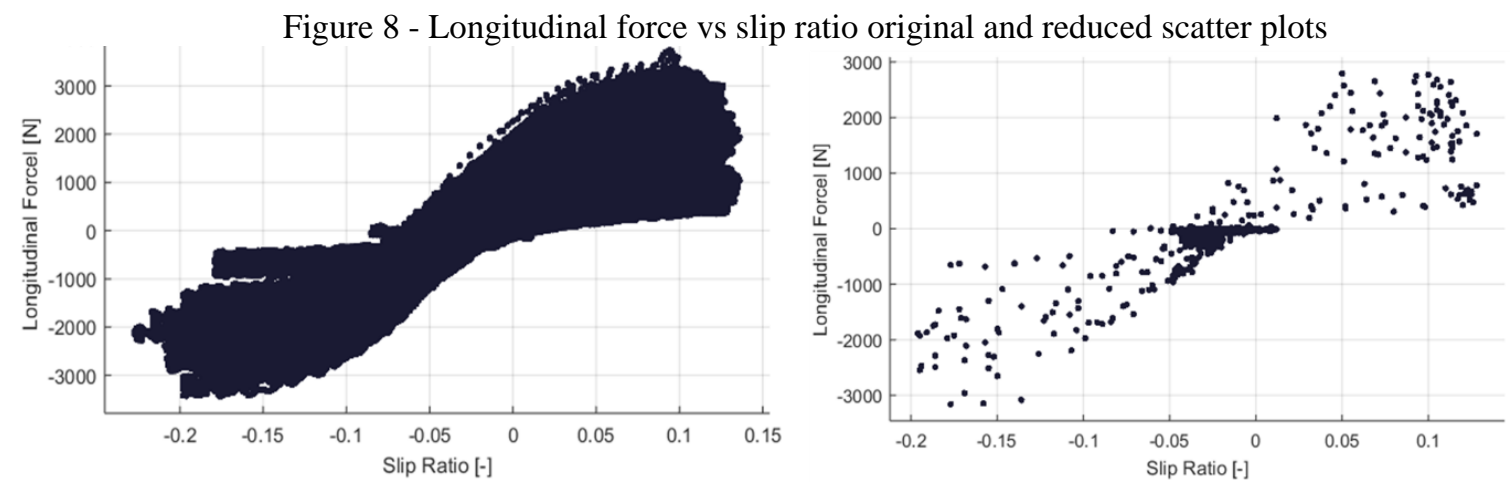

Data: "Formula SAE Tire Test Consortium (FSAE TTC)" and the "Calspan Tire Research Facility (TIRF)" 
Figure 9 - Camber vs pressure original and reduced scatter plots
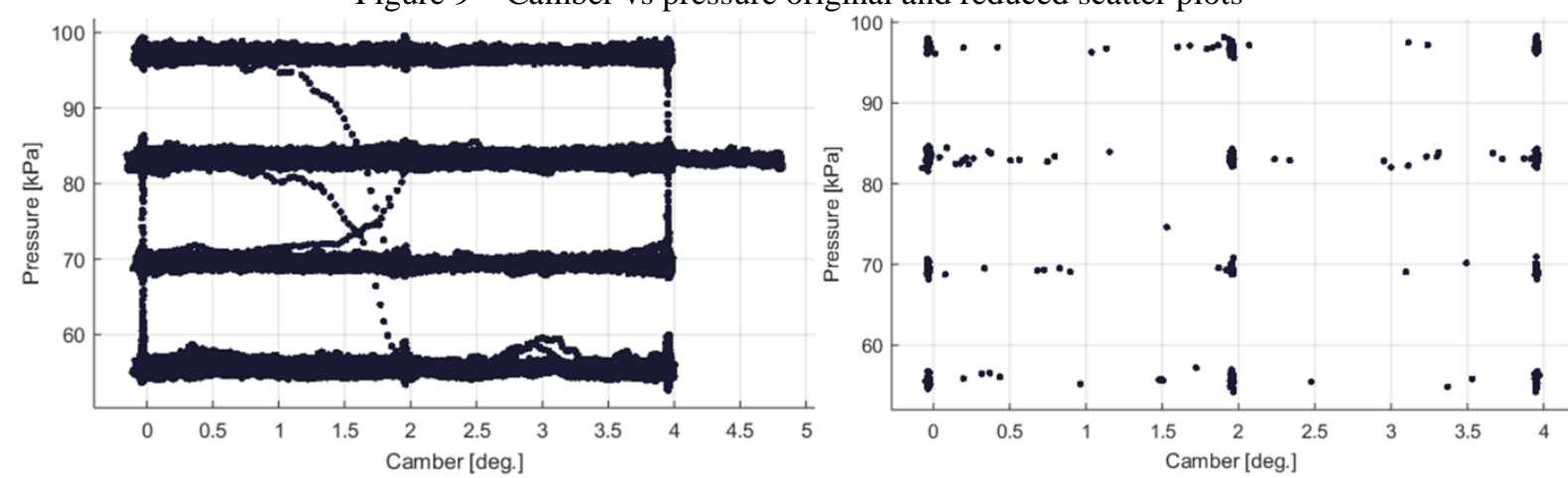

Data: "Formula SAE Tire Test Consortium (FSAE TTC)" and the "Calspan Tire Research Facility (TIRF)"

Figure 10 - Lateral force vs vertical load original and reduced scatter plots
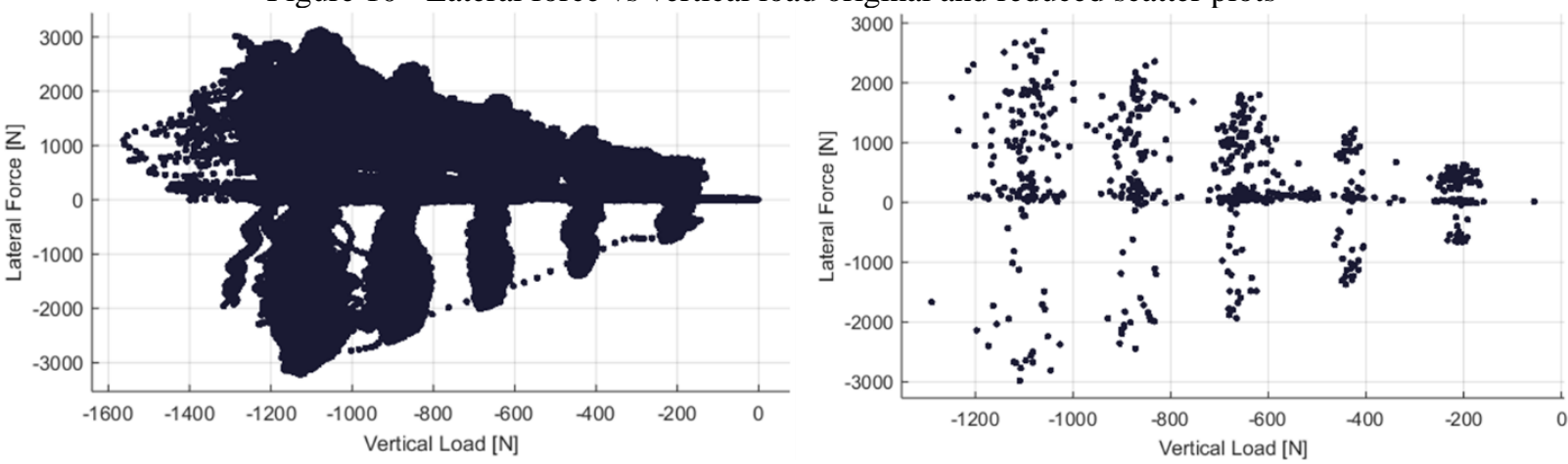

Data: "Formula SAE Tire Test Consortium (FSAE TTC)" and the "Calspan Tire Research Facility (TIRF)"

Figure 11 - Lateral force vs slip angle original and reduced scatter plots
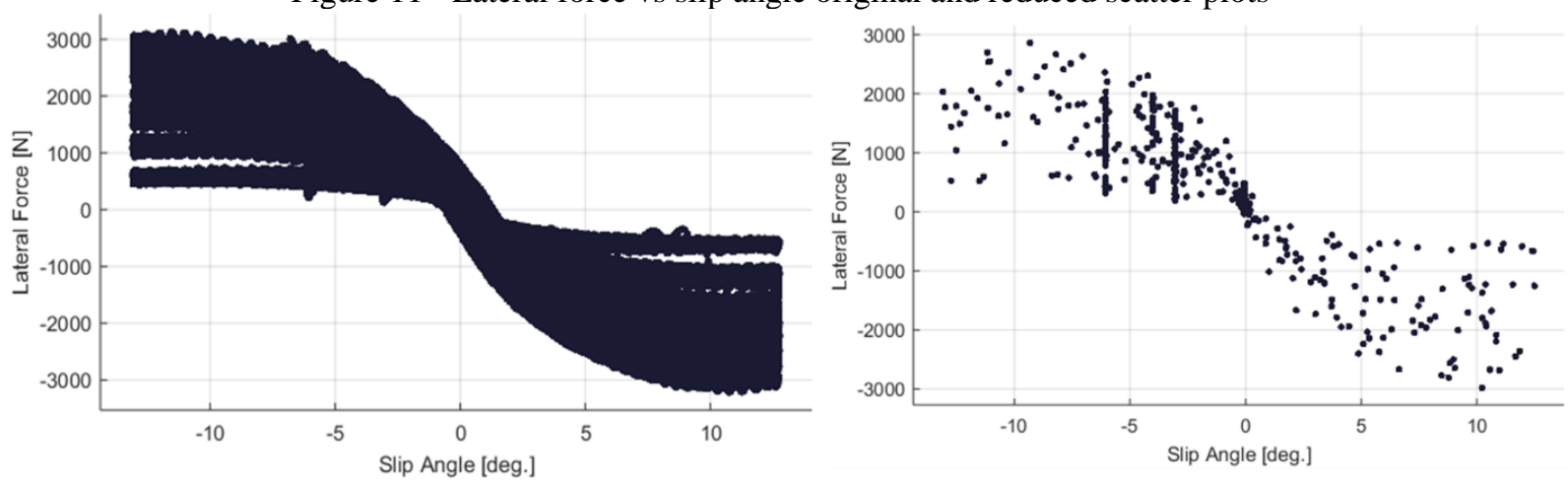

Data: "Formula SAE Tire Test Consortium (FSAE TTC)" and the "Calspan Tire Research Facility (TIRF)"

Figure 12 - Lateral force vs slip ratio original and reduced scatter plots
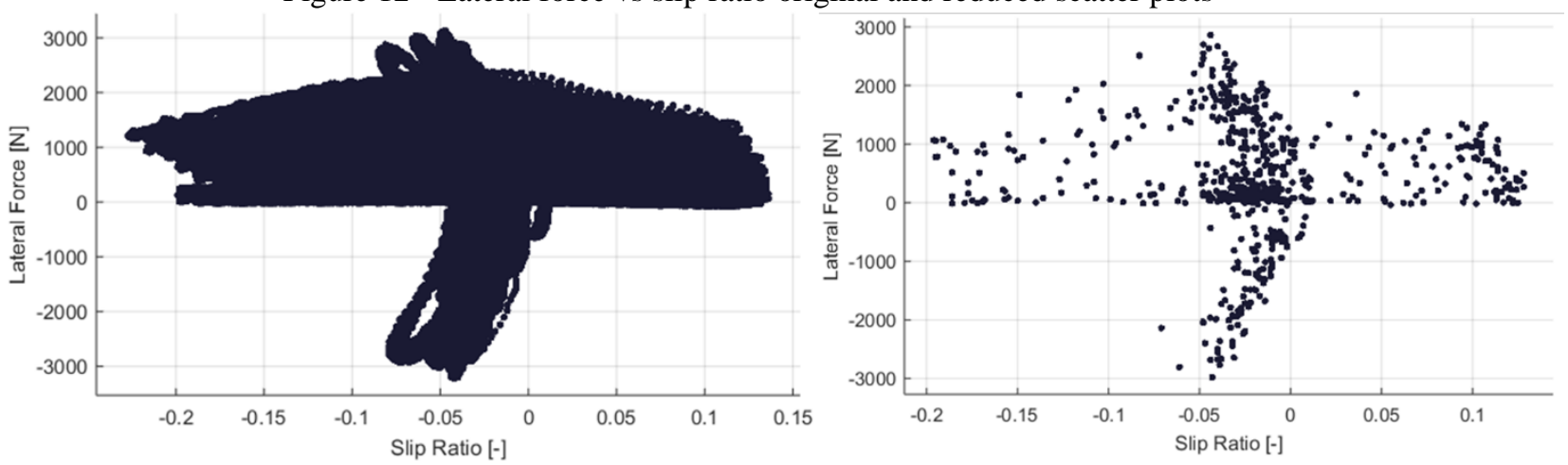

Data: "Formula SAE Tire Test Consortium (FSAE TTC)" and the "Calspan Tire Research Facility (TIRF)" 


\section{MODEL RESULTS AND ANALYSIS}

\subsection{Preliminary model quality analysis}

A multivariate Gaussian process regression model was created in ETAS ASCMO platform using the 800 training points selected. Figure 13 shows its user interface for illustrational purposes. The inputs are present in the lower bar and the outputs with its respective standard deviation values on the left bar. Black lines represent model predictions while the dashed lines show the size of a standard deviation. Finally, the gray dots are all the training points used to generate the model projected over the hyperplane defined by the current set of inputs.

Figure 13 - Leave-One-Out plot for modeled lateral and longitudinal forces

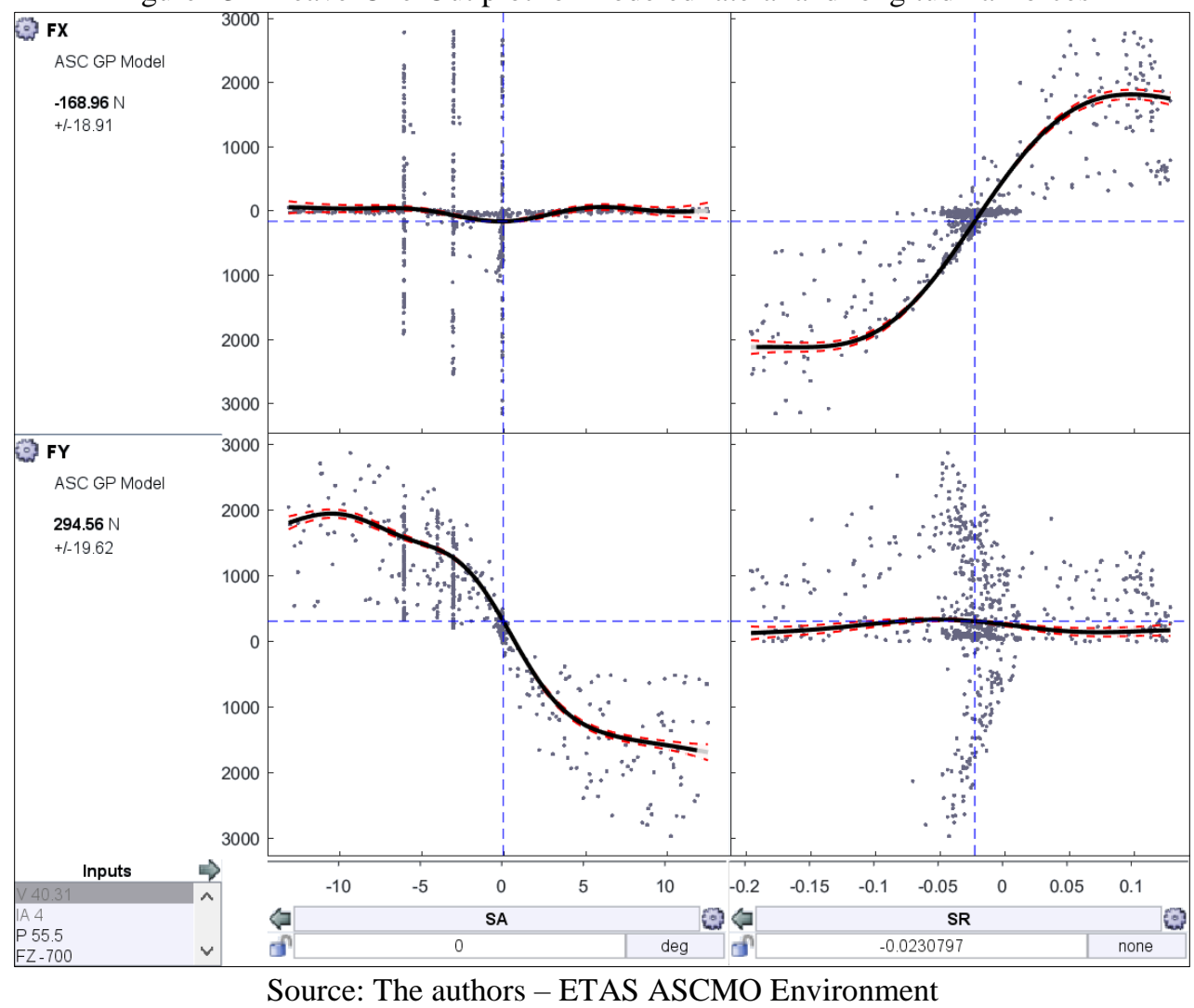

Before explicitly comparing the results to measurement data, global model quality was evaluated using the coefficient of determination $R^{2}$ and the root mean square error $R M S E$ as metrics:

$$
\begin{aligned}
& R M S E=\sqrt{\frac{S S R}{n}} \\
& R^{2}=1-\frac{S S R}{S S T}, \text { where }
\end{aligned}
$$

$S S R=\sum_{i=1}^{n}\left(x_{i, \text { pred }}-x_{i, \text { meas }}\right)^{2}-$ sum of squared residuals

$S S T=\sum_{i=1}^{n}\left(x_{i, \text { meas }}-\overline{x_{\text {meas }}}\right)^{2}$ - total sum of squares

$n=$ number of data points (training or test) under analysis 
The RMSE describes the variance to be expected (standard deviation) about the model: A second measurement falls less than 1 RMSE from the model prediction with a probability of $68 \%$ (with $95.5 \%<2$ RMSE, $99.7 \%<3$ RMSE, etc.). The coefficient of determination, $R^{2}$, is derived from the comparison of the variance that remains after the model training (SSR) with the variance concerning the mean value of all measuring data (SST) [6].

ETAS [6] suggests the following value ranges for evaluating $R^{2}$ :

- $0.0<R^{2}<0.5$ - The model is not suitable for reliable predictions.

- $0.6<R^{2}<0.8$ - The model is suited for qualitative predictions.

- $\quad 0.9<R^{2}<1.0$ - The model is very good and suitable for quantitative predictions.

ETAS [6] also comments on RMSE:

- At best, the RMSE can be as good as the experimental repeatability.

- Despite a good $R^{2}$, the RMSE can be too low, e.g. in case of a very large variation range of the modeled variable.

- Despite a low $R^{2}$, the RMSE can be good enough, e.g. if the modeled variable features only a minor variance over the input parameters of the model.

Gaussian process regression is particularly suited for leave-one-out cross validation techniques. In the leave-one-out method (LOO), $\mathrm{n}$ models, each with $\mathrm{n}-1$ training data, are formed. Afterwards, the model error of the one data point that was not involved in the model training is determined [6]. ASCMO implements this algorithm automatically. The results are presented in figure 14:

Figure 14 - Leave-One-Out plot for modelled lateral and longitudinal forces

FX (ASC GP)

RMSE: 74.1677, R2: 0.992006

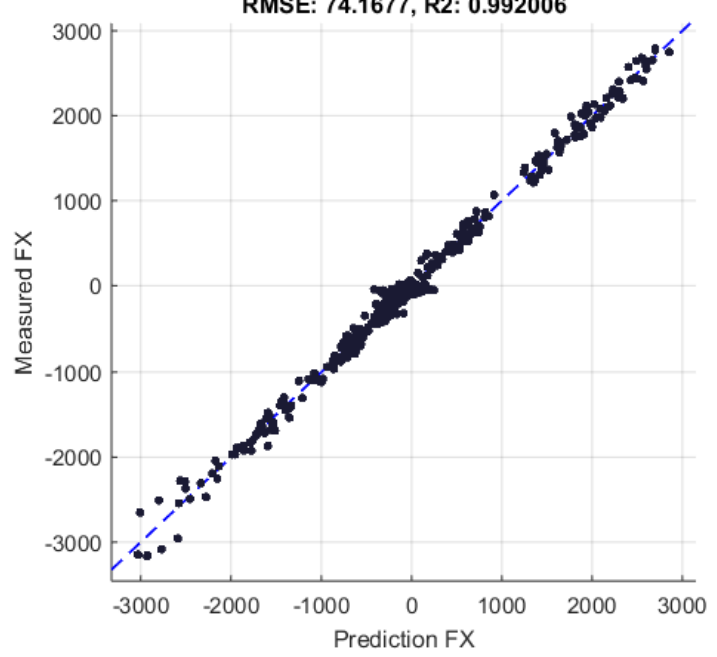

FY (ASC GP)

RMSE: 85.9743, R2: 0.991237

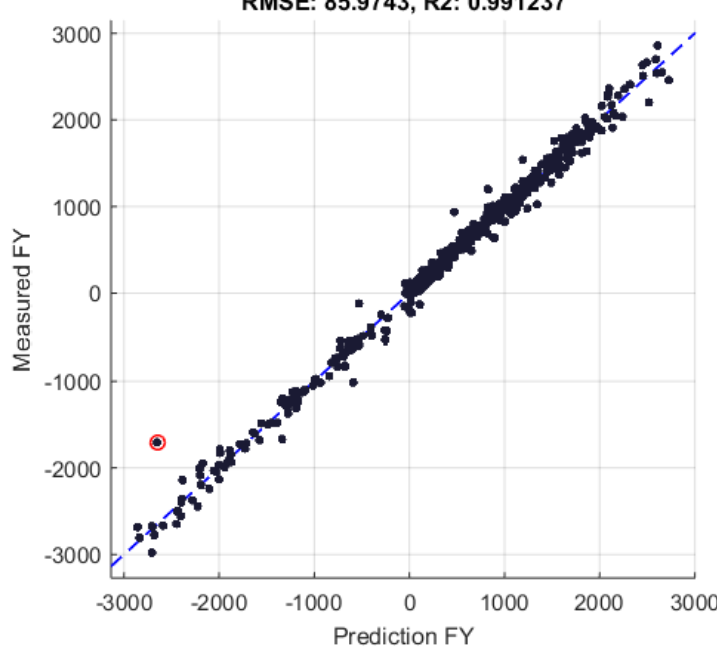

Source: The authors - ETAS ASCMO Environment 
The $R^{2}$ coefficient was found to be extremely close to the unity, indicating a possibly highquality model. The calculated RMSE, lower than 100, was judged adequate in face of longitudinal and lateral forces expected magnitude.

The same plot was generated comparing the model predictions with test data issuing good results: $R^{2} \cong 1$ and a low RMSE value:

Figure 15 -Model prediction versus measured data plot for lateral and longitudinal forces FX (ASC GP)

RMSE: 80.5915, R2: 0.990855

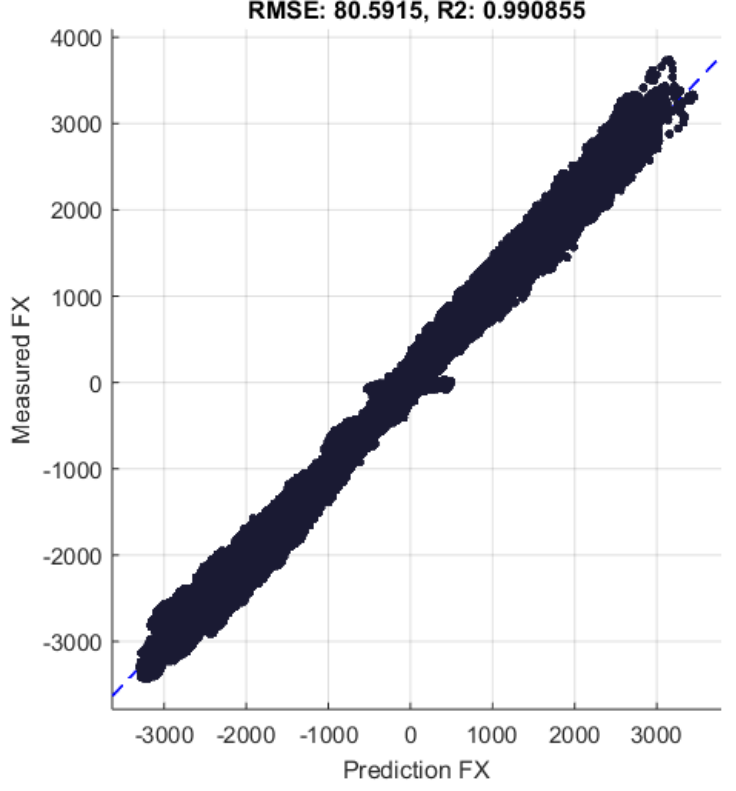
FY (ASC GP) RMSE: 80.3681, R2: 0.992534

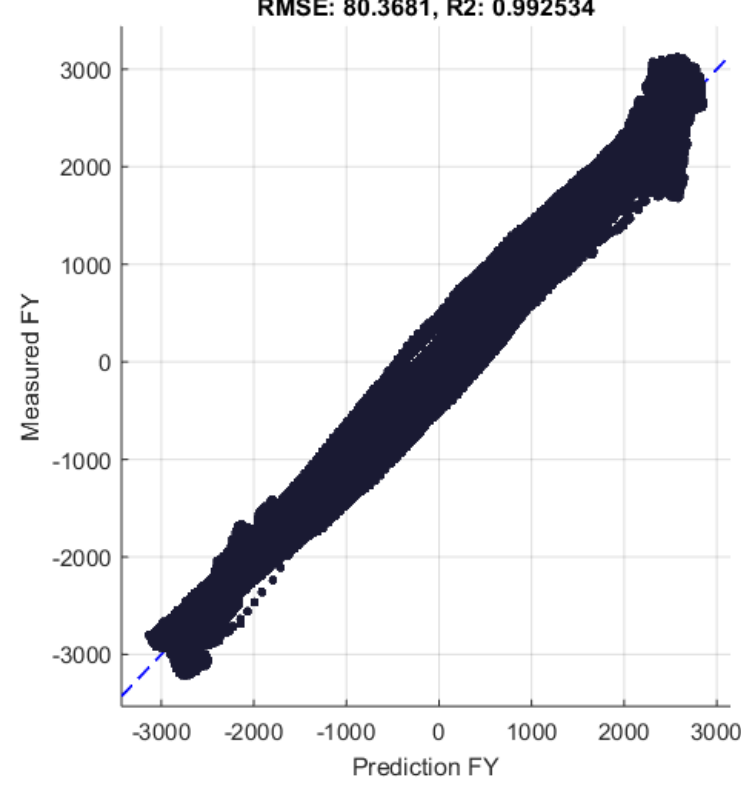

Source: The authors - ETAS ASCMO Environment

\subsection{Comparison with measured data}

In order to evaluate the validity and accuracy of the obtained model, some specific situations were chosen for the comparison between the prediction and the empirically measured data. Two classical and widely used plots for tire behavior analysis were used: Lateral Force versus Slip Angle and Longitudinal Force versus Slip Ratio, for multiple load values.

The first to be analyzed was the Lateral Force vs Slip Angle plot. The following procedure was adopted for the selection of the measured comparison points:

- The Slip Angle interval $\left(-12^{\circ}\right.$ to $\left.12^{\circ}\right)$ was uniformly divided into thirteen points;

- Pressure and Camber were kept in 12 psi and $0^{\circ}$, respectively;

- For each of the five Vertical Load regions used by FSAE TTC, shown in figure 16, an average value was extracted.

- Lateral Force values were obtained by averages made for all measure data points that met the combinations of parameters mentioned above. 
Thus, a total of 65 measured operating points was obtained. Using the same exact conditions, the five curves shown in the figure 16, below, were generated by the predictive model. Comparing the predicted values with those measured, the accuracy of the model is evident.

Figure 16 - Lateral Force versus Slip Angle for multiple loads - IA $=0$ deg, $\mathrm{P}=12 \mathrm{lbs}$

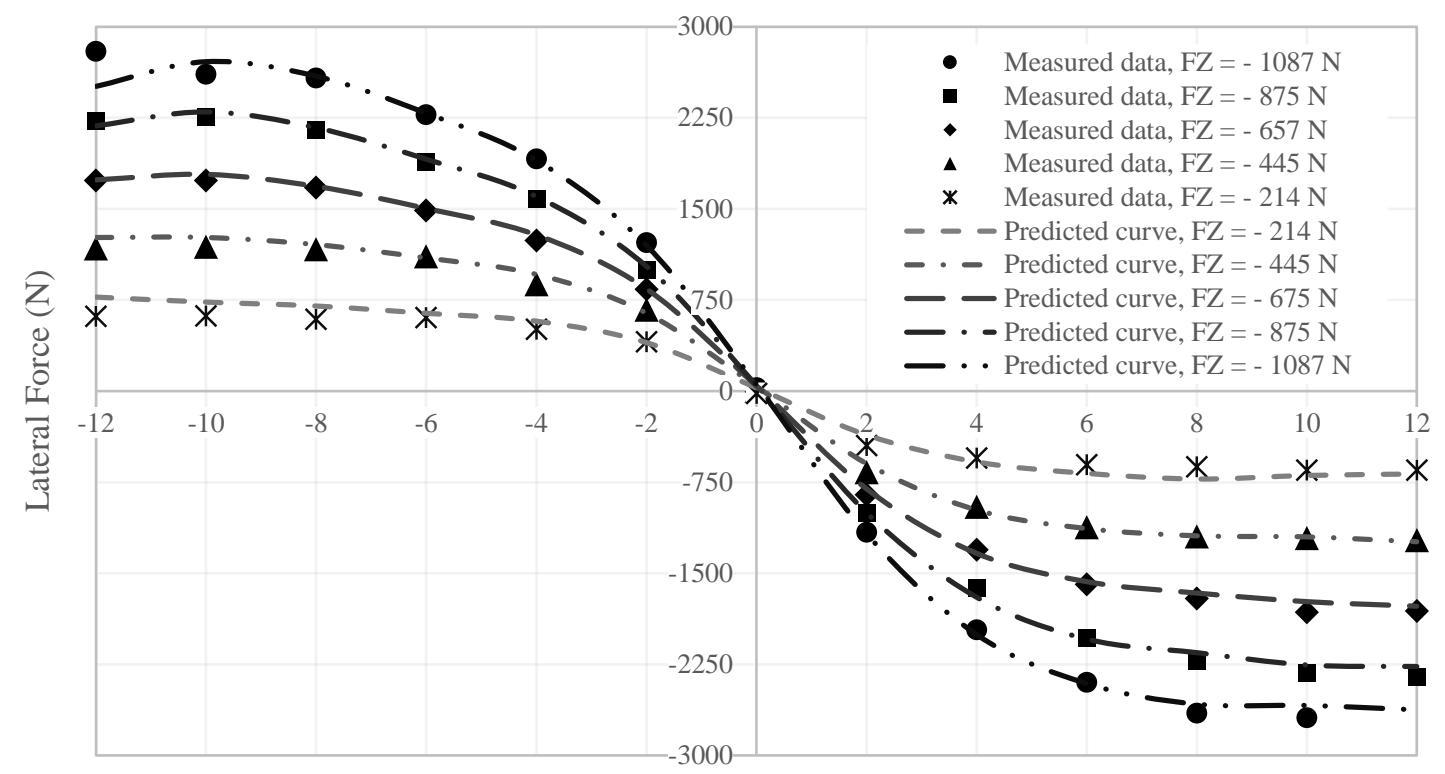

Slip Angle (deg.)

A similar analysis to the previous case was made using practically the same conditions, changing only the camber value from $0^{\circ}$ to $4^{\circ}$. This is an aggressive and unusual value for the tire used, which could cause difficulties to the mathematical model. The figure 17 shows a comparison between the measured and predicted data. It is possible to see that the model remains faithful to the test data, although it presents a slightly bigger error due to an unusual condition being analyzed.

Figure 17 - Lateral Force versus Slip Angle for multiple loads - IA = 4 deg, P = 12lbs

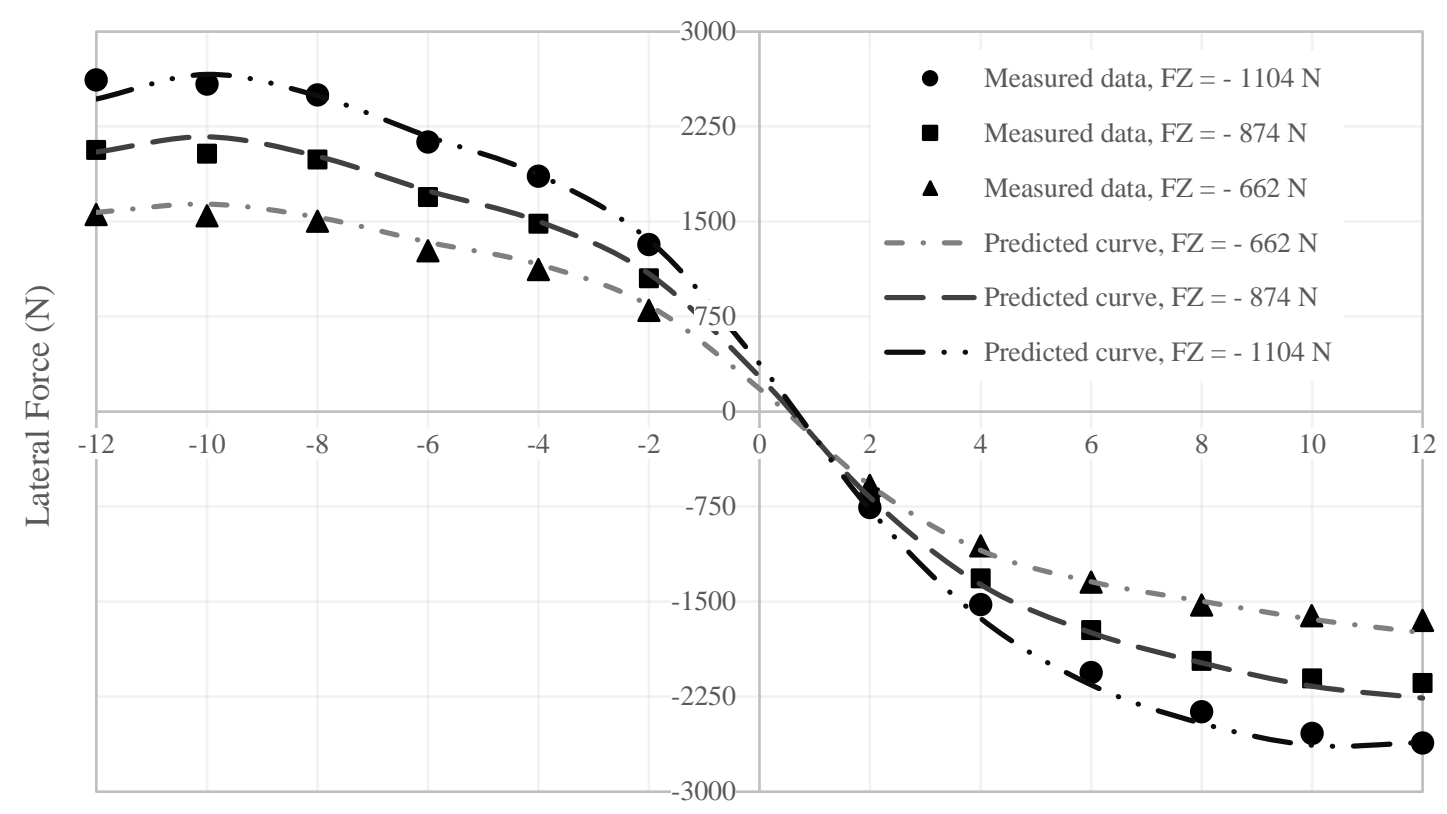

Slip Angle (deg.) 
The Longitudinal Force versus Slip Ratio plot analysis adopted the same procedure used for Lateral Force. However, in this case, the Slip Ratio was discretized at 13 points, in a range of 0,12 to $-0,20$. Camber, Pressure and Slip Angle were kept constant at $0^{\circ}, 12$ psi and $0^{\circ}$, respectively. Even though the Vertical Loads were chosen following the same procedures, the values found for the averages were slightly different from the first case.

Figure 18 shows a comparison between the measured and predicted data. In spite of presenting a slightly larger error, the model still closely resembles the real data. It is worth to mention that, as explained in section 2.3, the definition of Calspan for Slip Ratio is being used, which causes the asymmetry in relation to the vertical axis.

Figure 18 - Longitudinal Force versus Slip Ratio for multiple loads $-\mathrm{IA}=0 \mathrm{deg}, \mathrm{P}=12 \mathrm{lbs}$

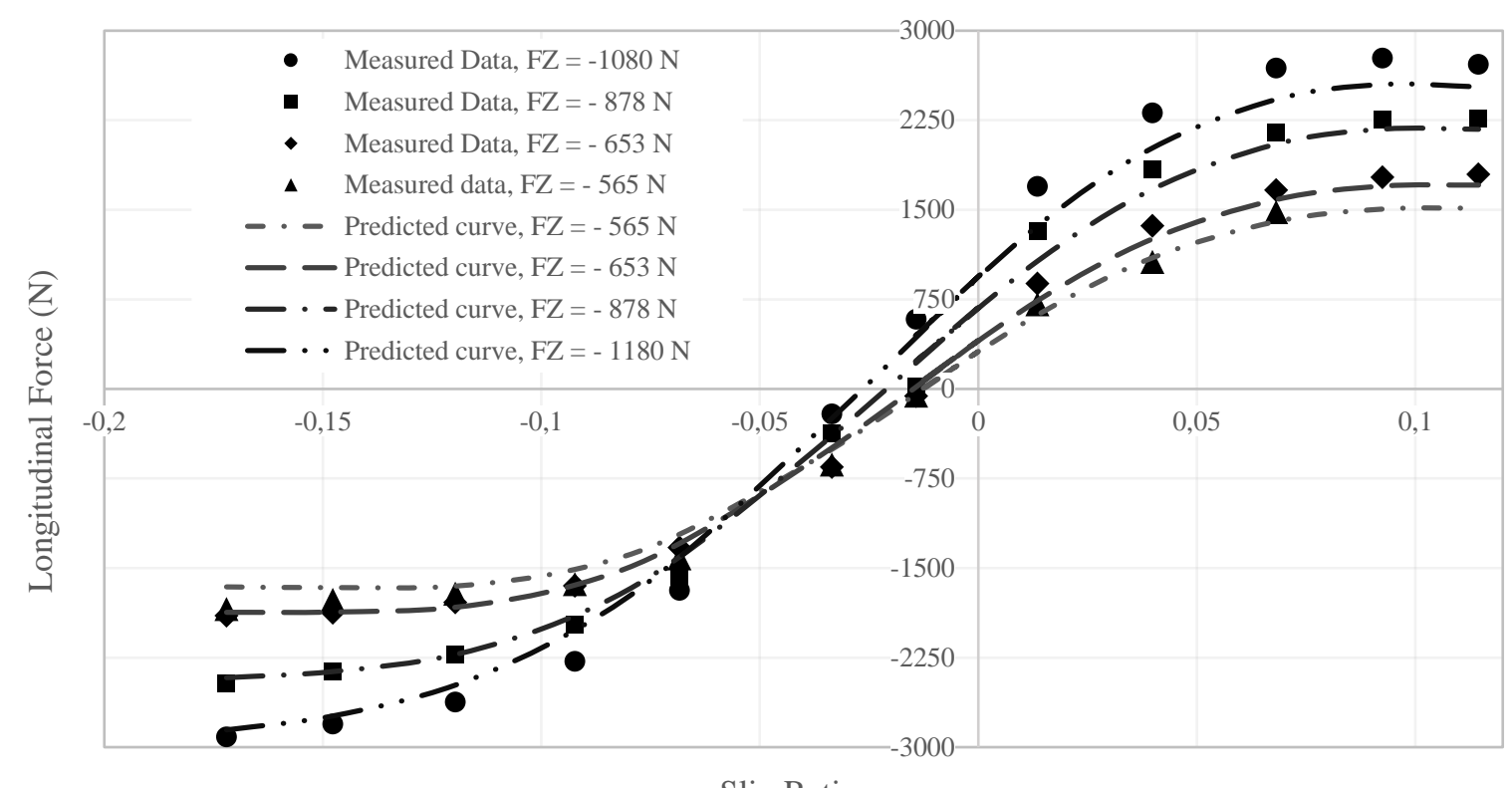

Slip Ratio

All curves shown above represent predicted results for tire behavior in the determined conditions. It is important to highlight the independence between test data and training data: no points used to train the model were present in the data used for validation.

Considering the enormous amount of measurement points available in test dataset and the reduced model evaluation time, the authors believe many other techniques could be devised and employed to validate de model systemically.

\subsection{Friction Ellipse}

Finally, a tire friction ellipse was constructed with model predictions. The external line on figure 19 is the limit line, which represents the maximum combined pair of forces possible to be generated by the tire. It was determined using ASCMO optimization algorithms, varying all input parameters within the model and them generating a Pareto frontier of optimal combinations for Lateral Force and Longitudinal Force. The remaining lines belong to an arbitrarily chosen operating condition that is common for FSAE vehicles: Vertical Load $=1150$ $\mathrm{N}$; Pressure $=12 \mathrm{psi}$; Camber $=0^{\circ} ; \mathrm{V}=25 \mathrm{mph}$. With the remaining input parameters fixed, Slip Angle is kept constant while Slip Ratio varies and vice versa. The procedure can be easily repeated for any other operating condition. 
Figure 19 - Friction Ellipse - Limit $\& \mathrm{Fz}=1150 \mathrm{~N}, \mathrm{IA}=0 \mathrm{deg}, \mathrm{P}=12 \mathrm{lbs}, \mathrm{V}=25 \mathrm{mph}$

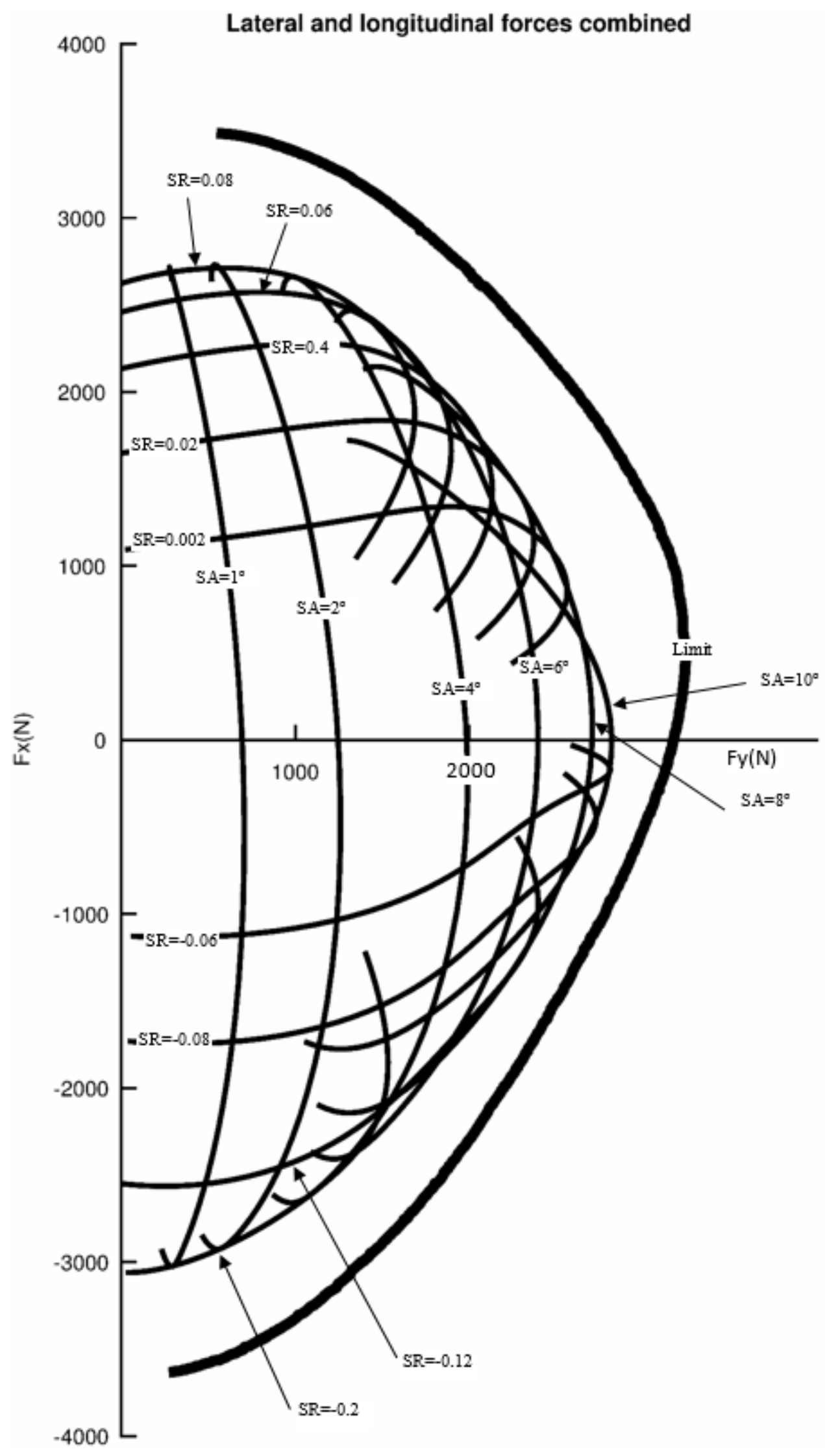




\section{CONCLUSION}

A Gaussian process model for tires in combined slip case was successfully elaborated using ETAS ASCMO platform combined with data from Formula SAE Tire Test Consortium generated at Calspan testing facility.

The mathematical framework used by ASCMO was presented in details and some of its aspects were highlighted: the model is continuous, it has an internal quality metric (simple local standard deviation estimation) and, although computationally consuming to train, is fast while making predictions as the outputs are described as a finite sum of kernel functions. Furthermore, once the model has been trained, any arbitrary combination of the available inputs can be analyzed without the need of a new training process.

The model was generated using 800 from an available universe 567.077 points. Its quality was initially evaluated via cross-validation using leave-one-out methods with good results for the root mean square error (RMSE) and the coefficient of determination $R^{2}$. These parameters were as well determined for test dataset (all data minus training data) with same satisfactory results.

Finally, the model was compared to measured points in three specific cases comprising combined slip angle and slip ratio interactions at different loads. Two different camber angles were tested in order to evaluate the model capabilities to cope with an arbitrary change in one of its input parameters. The results were found to be satisfactory.

- Lateral Force versus Slip Angle for multiple loads $-\mathrm{IA}=0 \mathrm{deg}, \mathrm{P}=12 \mathrm{lbs}$

- Lateral Force versus Slip Angle for multiple loads - IA = $4 \mathrm{deg}, \mathrm{P}=12 \mathrm{lbs}$

- Longitudinal Force versus Slip Ratio for multiple loads $-\mathrm{IA}=0 \mathrm{deg}, \mathrm{P}=12 \mathrm{lbs}$

To sum up, the method employed has shown itself to be efficient. From a simple analysis, such as Lateral Force vs Slip Angle plots, to complex evaluations, such as creating a friction ellipse for a specific set of input parameters, the model has worked well.

Amongst other modeling methods, both empirical and analytical, the method presented here gathers excellent adherence to the measured data, relative ease to obtaining the model and simplicity of use. Even analysis involving simultaneous variations on the various input parameters of the tires, which might be very complex to do have been successfully executed. Additional investigations for predictions on more complex parameters as tire moments and spring rate should be carried in the future in order to verify Gaussian process regression suitability for modelling a broader range of tire behavior.

\section{REFERENCES}

[1]SALVAGNI, Ronaldo B.; ALVES, Marcelo A. L.; BARBOSA, Roberto S.; "A GEOMETRICAL MODEL FOR TIRES UNDER VERTICAL LOADING", p. 1-15 . In: In Anais do XXI Simpósio Internacional de Engenharia Automotiva - SIMEA 2013. São Paulo: Blucher, 2014. ISSN 2357-7592, DOI 10.5151/engpro-simea-PAP2

[2] PACEJKA, H. B. (2006) - Tire and vehicle dynamics, 2nd Edition, SAE International, USA. 
[3] C. E. Rasmussen \& C. K. I. Williams, Gaussian Processes for Machine Learning, the MIT Press, 2006, (Available at http://www.gaussianprocess.org/gpml/chapters/RW.pdf)

[4] MILLIKEN, William F.; MILLIKEN, Douglas L. Race Car Vehicle Dynamics. Warrendale, PA. Society of Automotive Engineers, Inc. 1994.

[5] KASPRZAK, Edward M.; GENTZ, David. The Formula SAE Tire Test Consortium-Tire Testing and Data Handling. Society of Automotive Engineers, Inc. 2006.

[6] ETAS GmbH. ETAS ASCMO Static V5.2 - User's Guide, 2018. 